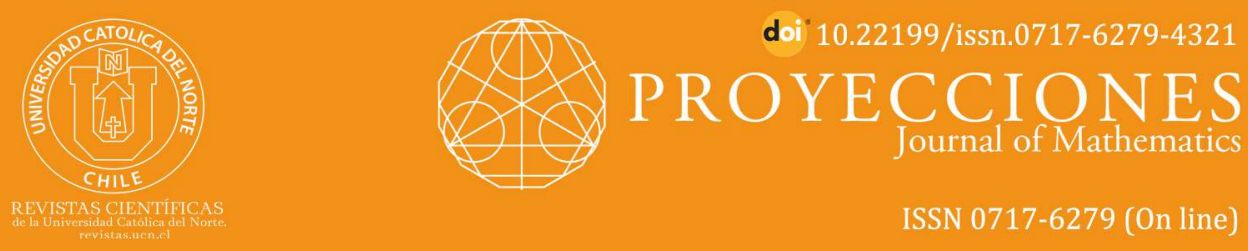

\title{
On some new paranormed sequence spaces defined by the matrix $(\hat{D})(\hat{r}, 0,0, \hat{s})$
}

Avinoy Paul ${ }^{1}$ @ orcid.org/0000-0002-4948-8647

${ }^{1}$ Cachar College, Dept. of Mathematics, Silchar, AS, India.

avinoypaul@gmail.com

Received: 07 July 2020 | Accepted: 25 December 2020

\section{Abstract:}

In this paper, we introduce some new paranormed sequence spaces and study some topological properties. Further, we determine $\alpha, \beta$ and $\gamma$-duals of the new sequence spaces and finally, we establish the necessary and sufficient conditions for characterization of matrix mappings.

Keywords: Paranorm sequence space; $\alpha$-dual; $\beta$-dual; $\gamma$-dual; Matrix characterization.

MSC (2020): 40A05, 40A25, 40C05, 40H05, 46A35, 47A10.

\section{Cite this article as (IEEE citation style):}

A. Paul, "On some new paranormed sequence spaces defined by the matrix $(\hat{D})(\hat{r}, 0,0, \hat{S})$ ", Proyecciones (Antofagasta, On line), vol. 40, no. 3, pp. 779-796, 2021, doi: 10.22199/issn.0717-6279-4321

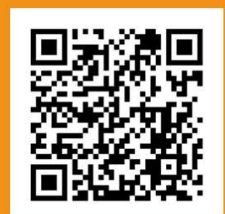

Article copyright: (C) 2021 Avinoy Paul. This is an open access article distributed under the terms of the Creative Commons License, which permits unrestricted use and distribution provided the original author and source are credited. 


\section{Introduction}

The idea to construct a new sequence space by means of the matrix domain of a particular limitation method has recently been employed by Altay and Basar[3, 4], Malkowsky and Savas[17], Basar et al.[7], Kirisci and Basar[11], $\mathrm{Ng}$ and Lee[18], Sönmez[21] and many more. Moreover, Altay and Basar $[1,2]$, Malkowsky[16] and Aydin and Basar[6] have employed on to construct new paranormed sequence spaces by means of the domain of some infinite matrices. The domain of generalized difference matrix $B(r, s)$ on some Maddox's spaces was studied by Aydin and Altay [5]. More recently, domain of the double sequential band matrix $B(\tilde{r}, \tilde{s})$ on some Maddox's spaces was studied by $\ddot{O}$ zger and Basar[19].

\section{Preliminaries}

Throughout the paper we denote $w, \ell_{\infty}, c, c_{0}$ and $\ell_{p}$ be the space of all, bounded, convergent, null and $p$-absolutely summable sequences respectively. Also, $b s, c s$ and $\ell_{1}$ denote the spaces of all bounded, convergent and absolutely convergent series respectively.

Let $X$ and $Y$ be two sequence spaces and $B=\left(b_{n k}\right)$ be an infinite matrix of real or complex numbers $b_{n k}$, where $n, k \in \mathbf{N}=\{1,2,---\}$. Then, we say that $B$ defines a matrix mapping from $X$ into $Y$, denoted by $B: X \rightarrow Y$, if for every sequence $x=\left(x_{n}\right) \in X$, the sequence $B x=(B x)_{n}$ is in $Y$ where,

$$
(B x)_{n}=\sum_{k=1}^{\infty} b_{n k} x_{k},(n \in \mathbf{N a n d} x \in X),
$$

provided the right hand side converges for every $n \in \mathbf{N}$ and $x \in X$.

If $\mu$ is a normed sequence space, we write $D_{\mu}(B)$ for $x \in w$ for which the sum in (2.1) converges in the norm of $\mu$. We write $(\lambda, \mu)=\left\{B: \lambda \subset D_{\mu}(B)\right\}$ for the space of those matrices which send the whole of the sequence space $\lambda$ into the sequence space $\mu$ in this sense.

The sequence space $\lambda_{B}=\left\{x=\left(x_{k}\right) \in w: B x \in \lambda\right\}$ is called the domain of an infinite matrix $B$ in a sequence space $\lambda$. One can easily verify that the sequence spaces $\lambda_{B}$ and $\lambda$ are linearly isomorphic when $B$ is triangle. 
A paranormed space $(X, g)$ is a topological linear space in which the topology is given by paranorm $g$, a real sub-additive function on $X$ such that $g(\theta)=0, g(x)=g(-x)$ and scalar multiplication is continuous means that $\lambda_{n} \rightarrow \lambda, x_{n} \rightarrow x$ imply $\lambda_{n} x_{n} \rightarrow \lambda x$, for scalars $\lambda$ and vectors $x$.

We consider $\left(p_{k}\right)$ is a bounded sequence of positive real numbers with supp $_{k}=H$ and $M=\max \{1, H\}$. Throughout we assume $p_{k}^{-1}+\left(p_{k}\right)^{-1}=1$ provided $0<$ infp $p_{k} \leq H<\infty$.

Throughout $\mathbf{C}$ denotes the complex field.

Maddox [13] define the following sequence spaces:

$$
\begin{aligned}
& \ell_{\infty}(p)=\left\{x=\left(x_{k}\right) \in w: \sup _{k \in \mathbf{N}}\left|x_{k}\right|^{p_{k}}<\infty\right\}, \\
& c(p)=\left\{x=\left(x_{k}\right) \in w: \exists l \in \mathbf{C}, \ni \lim _{k \rightarrow \infty}\left|x_{k}-l\right|^{p_{k}}=0\right\}, \\
& c_{0}(p)=\left\{x=\left(x_{k}\right) \in w: \ni \lim _{k \rightarrow \infty}\left|x_{k}\right|^{p_{k}}=0\right\}, \\
& \ell(p)=\left\{x=\left(x_{k}\right) \in w: \sum_{k}\left|x_{k}\right|^{p_{k}}<\infty\right\},\left(0<p_{k}<\infty\right) .
\end{aligned}
$$

If $p \in \ell_{\infty}$ then (Maddox[14], Theorem 6]) $c_{0}(p)$ and $c(p)$ are complete paranormed sequence spaces paranormed by $g_{1}(x)=\sup _{k \in \mathbf{N}}\left|x_{k}\right|^{\frac{p_{k}}{M}}$. Also $\ell(p)$ is a complete paranormed sequence space paranormed by $g_{1}$ if and only if $i n f p_{k}>0$. Further, $\ell(p)$ is complete paranormed sequence space paranormed by $g_{2}(x)=\left(\sum_{k}\left|x_{k}\right|^{p_{k}}\right)^{\frac{1}{M}}$.

Let, $\hat{r}=\left(r_{k}\right)$ and $\hat{s}=\left(s_{k}\right)$ are convergent sequences whose entries either constants or distinct non-zero numbers then we define the matrix $\hat{D}(\hat{r}, 0,0, \hat{s})$ as follows: $\hat{D}(\hat{r}, 0,0, \hat{s})=\left[d_{n k}(r, s)\right]$ where,

$$
d_{n k}(r, s)= \begin{cases}r_{k}, & (k=n) \\ s_{k}, & (k=n-3) \\ 0, & \text { otherwise }\end{cases}
$$

for all $k, n \in \mathbf{N}$. 


\section{Some new paranormed sequence spaces and their topolog- ical properties}

We define the sequence spaces $\ell_{\infty}(\hat{D}, p), c(\hat{D}, p)$ and $c_{0}(\hat{D}, p)$ as the set of sequences whose transforms are in the spaces $\ell_{\infty}(p), c(p)$ and $c_{0}(p)$ respectively, that is-

$$
\begin{aligned}
& \ell_{\infty}(\hat{D}, p)=\left\{x=\left(x_{k}\right): \sup _{k \in \mathbf{N}}\left|r_{k} x_{k}+s_{k-3} x_{k-3}\right|^{p_{k}}<\infty\right\}, \\
& c(\hat{D}, p)=\left\{x=\left(x_{k}\right): \exists l \in \mathbf{C}, \ni \lim _{k \rightarrow \infty}\left|r_{k} x_{k}+s_{k-3} x_{k-3}-l\right|^{p_{k}}=0\right\}, \\
& c_{0}(\hat{D}, p)=\left\{x=\left(x_{k}\right): \lim _{k \rightarrow \infty}\left|r_{k} x_{k}+s_{k-3} x_{k-3}\right|^{p_{k}}=0\right\} .
\end{aligned}
$$

Theorem 3.1 The sequence spaces $\ell_{\infty}(\hat{D}, p), c(\hat{D}, p)$ and $c_{0}(\hat{D}, p)$ are the complete linear metric spaces paranormed by $g$, defined by $g(x)=\sup _{k \in \mathbf{N}} \mid r_{k} x_{k}+$ $\left.s_{k-3} x_{k-3}\right|^{p_{k}}$.

Proof: We proof the result only for the space $c_{0}(\hat{D}, p)$ to avoid the repetition of the similar statements for other given spaces. One can easily prove that $c_{0}(\hat{D}, p)$ is a linear space with co-ordinate wise addition and scalar multiplication since $\hat{D}(\hat{r}, 0,0, \hat{s})$ is a triangle matrix and $c_{0}(p)$ is a linear space.

It is clear that $g(\theta)=0, g(x) \geq 0$ for all $x \in c_{0}(\hat{D}, p)$ and $g(-x)=g(x)$. Consider any sequence $\left\{x^{n}\right\}$ of points of $c_{0}(\hat{D}, p)$ such that $g\left(x^{n}-x\right) \rightarrow 0$ as $n \rightarrow \infty$ and $\left(\beta_{n}\right)$ is a sequence of scalars with $\beta_{n} \rightarrow \beta$ as $n \rightarrow \infty$.

Now, $\left\{g\left(x^{n}\right)\right\}$ is bounded sequence since $g\left(x^{n}\right) \leq g(x)+g\left(x^{n}-x\right)$ holds by subadditivity of the function $g$. Again, we have,

$$
\begin{gathered}
\left(\beta_{n} x^{n}-\beta x\right)=\sup _{k \in \mathbf{N}}\left|r_{k}\left(\beta_{n} x_{k}^{n}-\beta x_{k}\right)+s_{k-3}\left(\beta_{n} x_{k-3}^{n}-\beta x_{k-3}\right)\right|^{\frac{p_{k}}{M}} \\
\leq\left|\beta_{n}-\beta\right| g\left(x^{n}\right)+|\beta| g\left(x^{n}-x\right)
\end{gathered}
$$

which tends to zero as $n \rightarrow \infty$.

Thus, scalar multiplication is continuous and hence $c_{0}(\hat{D}, p)$ is a paranormed sequence space.

Next, let $\left\{x^{i}\right\}$ be a Cauchy sequence in $c_{0}(\hat{D}, p)$, where $x^{i}=\left\{x_{1}^{(i)}, x_{2}^{(i)}, x_{3}^{(i)},---\right\}$.

Then, by the definition of Cauchy sequence, for a given $\varepsilon>0$, there exists 
a positive integer $n_{0}$ depending on $\varepsilon$ such that $g\left(x^{i}-x^{j}\right)<\varepsilon$ for all $i, j \geq 0$.

Now, using the definition of $g$ for each fixed $k \in \mathbf{N}$,

$$
\left|\left\{\hat{D}(\hat{r}, 0,0, \hat{s}) x^{i}\right\}_{k}-\left\{\hat{D}(\hat{r}, 0,0, \hat{s}) x^{j}\right\}_{k}\right|
$$

$$
\leq \sup _{k \in \mathbf{N}}\left|\left\{\hat{D}(\hat{r}, 0,0, \hat{s}) x^{i}\right\}_{k}-\left\{\hat{D}(\hat{r}, 0,0, \hat{s}) x^{j}\right\}_{k}\right|^{\frac{p_{k}}{M}}<\varepsilon
$$

for every $i, j \in n_{0}$, which leads us to the fact that

$$
\left\{\left\{\hat{D}(\hat{r}, 0,0, \hat{s}) x^{1}\right\}_{k},\left\{\hat{D}(\hat{r}, 0,0, \hat{s}) x^{2}\right\}_{k},\left\{\hat{D}(\hat{r}, 0,0, \hat{s}) x^{3}\right\}_{k},---\right\}
$$

is a Cauchy sequence of complex numbers and is convergent for each $k \in \mathbf{N}$.

Suppose, for each fixed $\mathrm{k}$,

$$
\left\{\hat{D}(\hat{r}, 0,0, \hat{s}) x^{i}\right\}_{k} \rightarrow(\hat{D}(\hat{r}, 0,0, \hat{s}) x)_{k}
$$

as $i \rightarrow \infty$.

Now, define the sequence

$$
\left\{(\hat{D}(\hat{r}, 0,0, \hat{s}) x)_{1},(\hat{D}(\hat{r}, 0,0, \hat{s}) x)_{2},(\hat{D}(\hat{r}, 0,0, \hat{s}) x)_{3},---\right\}
$$

From, the relation (3.1) with $j \rightarrow \infty$ we have,

$$
\sup _{k \in \mathbf{N}}\left|\left\{\hat{D}(\hat{r}, 0,0, \hat{s}) x^{i}\right\}_{k}-\{\hat{D}(\hat{r}, 0,0, \hat{s}) x\}_{k}\right|^{\frac{p_{k}}{M}}<\varepsilon
$$

for every fixed $k \in \mathbf{N}$.

Now, $x^{i}=\left\{x_{k}^{i}\right\} \in c_{0}(\hat{D}, p)$ implies

$$
\left|\left\{\hat{D}(\hat{r}, 0,0, \hat{s}) x^{i}\right\}_{k}\right|^{\frac{p_{k}}{M}}<\varepsilon,
$$

for each $k \in \mathbf{N}$.

Thus, by using relation (3.2) we have $\left|\{\hat{D}(\hat{r}, 0,0, \hat{s}) x\}_{k}\right|^{\frac{p_{k}}{M}}$ $\leq\left|\{\hat{D}(\hat{r}, 0,0, \hat{s}) x\}_{k}-\left\{\hat{D}(\hat{r}, 0,0, \hat{s}) x^{i}\right\}_{k}\right|^{\frac{p_{k}}{M}}+\left.\left\{\hat{D}(\hat{r}, 0,0, \hat{s}) x^{i}\right\}_{k}\right|^{\frac{p_{k}}{M}}<2 \varepsilon$

Hence, the sequence $\{\hat{D}(\hat{r}, 0,0, \hat{s}) x\}$ belongs to $c_{0}(p)$. Since, $\left\{x^{i}\right\}$ is an arbitrary Cauchy sequence, the space $c_{0}(\hat{D}, p)$ is complete. This completes 
the proof.

It is well known that the matrix domain $\lambda_{B}$ of a sequence space $\lambda$ has a basis whenever $B=\left(b_{n k}\right)$ is a triangle. (Jarrah and Malkowsky [10], Remark 2.4), we have

Proposition 3.2 Let $\gamma_{k}=\{\hat{D}(\hat{r}, 0,0, \hat{s}) x\}$ and $0<p_{k} \leq M<\infty$ for all $k \in \mathbf{N}$. Define the sequences $f=\left(f_{n}\right)$ and $d^{(k)}=\left\{d_{n}^{(k)}(\hat{r}, \hat{s})\right\}_{n \in \mathbf{N}}$ of the elements of the space $c_{0}(\hat{D}, p)$ by

$$
d_{n}^{(k)}(\hat{r}, \hat{s})=\left\{\begin{array}{l}
\text { For fixed } n, k \text { and for } j \in \mathbf{N} \\
d_{n+3 j}^{(k)}=\frac{(-1)^{n-k} s_{k} s_{k+3}---s_{n-3}}{r_{k} r_{k+3}--r_{n}} \quad n \geq k, \text { taking } s_{-t}=1 \\
0, \quad \text { otherwise. }
\end{array}\right.
$$

$f_{n}=\sum_{k=1}^{n} d_{n}^{(k)}(\hat{r}, \hat{s})$. Then, the following statements hold:

(i) The sequence $\left\{d^{(k)}\right\}$ is a basis for $c_{0}(\hat{D}, p)$ and any $x \in c_{0}(\hat{D}, p)$ has a unique representation of the form $x=\sum_{k} \gamma_{k} d^{(k)}$.

(ii) The set $\left\{f, d^{(k)}\right\}$ is a basis for $c(\hat{D}, p)$ and any $x \in c(\hat{D}, p)$ has a unique representation of the form $x=\eta f+\sum_{k}\left(\gamma_{k}-\eta\right) d^{(k)}$. where $\eta=$ $\lim _{k \rightarrow \infty}\{\hat{D}(\hat{r}, 0,0, \hat{s}) x\}_{k}$.

\section{Duals of the sequence spaces}

The idea of dual sequence space was introduced by Köthe and Toeplitz [12]. Then, Maddox, [15], generalized this notion to $-X$ valued sequence classes where $X$ is a Banach space. Further, Chandra Tripathy[8] studied on generalized Köthe-Toeplitz duals of some sequence spaces.

The set $S(\lambda, \mu)$ is defined by

$$
S(\lambda, \mu)=\left\{z=\left(z_{k}\right) \in w: x z \in \mu, \forall x=\left(x_{k}\right) \in \lambda\right\}
$$

is called the multiplier space of the spaces $\lambda$ and $\mu$. One can easily observe for a sequence space $\gamma$ with $\lambda \supset \gamma \supset \mu$ that the inclusions $S(\lambda, \mu) \subset S(\gamma, \mu)$ and $S(\lambda, \mu) \subset S(\lambda, \gamma)$ hold. With the notation (4.1), the $\alpha, \beta$ and $\gamma$ duals of a sequence space $\lambda$, which are respectively denoted by $\lambda^{\alpha}, \lambda^{\beta}$ and $\lambda^{\gamma}$ are defined by $\lambda^{\alpha}=S\left(\lambda, \ell_{1}\right), \lambda^{\beta}=S(\lambda, c s)$ and $\lambda^{\gamma}=S(\lambda, b s)$. 
Define the sequence $y=\left(y_{k}\right)$ which will be frequently used by $\hat{D}(\hat{r}, 0,0, \hat{s})$ transform of a sequence $x=\left(x_{k}\right)$, that is $y_{k}=\{\hat{D}(\hat{r}, 0,0, \hat{s}) x\}_{k}=r_{k} x_{k}+s_{k-3} x_{k-3}$ for $k \in \mathbf{N}$, taking

$$
x_{-3}=x_{-2}=x_{-1}=0
$$

Throughout we denote $N_{m}$ be the collection of those integers which are greater than or equal to $m \in \mathbf{N}$ and also $\wp$ denote the collection of all finite subsets of $\mathbf{N}$.

Theorem 4.1 Define the sets $T_{i}(p), i \in\{1,2,3,---, 7\}$ as follows:

(i) $T_{1}(p)=\bigcup_{S>1}\left\{a=\left(a_{k}\right) \in w: \sup _{K \in \wp} \sum_{n}\left|\sum_{k \in K} d_{n}^{(k)}(\hat{r}, \hat{s}) a_{n}\right| S^{\frac{-1}{p_{k}}}<\right.$ $\infty\}$,

(ii) $T_{2}(p)=\bigcup_{S>1}\left\{a=\left(a_{k}\right) \in w: \sum_{k}\left|\sum_{i=k}^{n} \frac{(-1)^{i} s_{k} s_{k+3}---s_{i-3}}{r_{k} r_{k+3}---r_{i}} a_{i}\right| S^{\frac{-1}{p_{k}}}<\right.$ $\infty\}$,

(iii) $T_{3}(p)=\bigcup_{S>1}\left\{a=\left(a_{k}\right) \in w:\left(\sum_{i=k}^{n} \frac{(-1)^{i} s_{k} s_{k+3}---s_{i-3}}{r_{k} r_{k+3}--r_{i}} a_{i} S^{\frac{-1}{p_{k}}}\right) \in \ell_{\infty}\right\}$,

(iv) $T_{4}(p)=\bigcup_{S>1}\left\{a=\left(a_{k}\right) \in w: \sup _{n \in \mathbf{N}} \sum_{k}\left|\sum_{i=k}^{n} \frac{(-1)^{i} s_{k} s_{k+3}---s_{i-3}}{r_{k} r_{k+3}---r_{i}} a_{i}\right| S^{\frac{-1}{p_{k}}}<\right.$ $\infty\}$,

(v) $T_{5}(p)=\bigcap_{S>1}\left\{a=\left(a_{k}\right) \in w: \sup _{K \in \wp} \sum_{n}\left|\sum_{k \in K} d_{n}^{(k)}(\hat{r}, \hat{s}) a_{n}\right| S^{\frac{-1}{p_{k}}}<\right.$ $\infty\}$

(vi) $T_{6}(p)=\bigcap_{S>1}\left\{a=\left(a_{k}\right) \in w: \sum_{k}\left|\sum_{i=k}^{n} \frac{(-1)^{i} s_{k} s_{k+3}---s_{i-3}}{r_{k} r_{k+3}---r_{i}} a_{i}\right| S^{\frac{-1}{p_{k}}}<\right.$ $\infty\}$,

(vii) $T_{7}(p)=\left\{a=\left(a_{k}\right) \in w: \sum_{n}\left|\sum_{k} d_{n}^{(k)}(\hat{r}, \hat{s}) a_{n}\right|<\infty\right\}$.

Then we have, $\left[c_{0}(\hat{D}, p)\right]^{\alpha}=T_{1}(p),\left[c_{0}(\hat{D}, p)\right]^{\beta}=T_{2}(p) \cap T_{3}(p),\left[c_{0}(\hat{D}, p)\right]^{\gamma}=$ $T_{4}(p)$,

$[c(\hat{D}, p)]^{\alpha}=T_{1}(p) \cap T_{7}(p),[c(\hat{D}, p)]^{\beta}=T_{2}(p) \cap T_{3}(p) \cap c s,[c(\hat{D}, p)]^{\gamma}=$ $T_{4}(p) \cap b s$,

$$
\left[\ell_{\infty}(\hat{D}, p)\right]^{\alpha}=T_{5}(p), \quad\left[\ell_{\infty}(\hat{D}, p)\right]^{\beta}=T_{6}(p) \cap c s, \quad\left[\ell_{\alpha}(\hat{D}, p)\right]^{\gamma}=T_{6}(p) .
$$


Proof : To avoid repetition and similar arguments we give the proof only for the sequence space $c_{0}(\hat{D}, p)$.

Let $f=\left(f_{n}\right) \in w$ and define the matrix $A=\left(a_{n k}\right)$ via the sequence $f=\left(f_{n}\right)$ by

$$
n k=\left\{\begin{array}{c}
{ }_{n}^{k}(\hat{r}, \hat{s}) f_{n}, \quad(1 \leq k \leq n) \\
0, \quad(k>n)
\end{array}\right.
$$

Using the relation (4.2), one can derive by straight forward calculation that

$f_{n} x_{n}=f_{n}\left\{\hat{D}^{-1}(\hat{r}, 0,0, \hat{s}) y\right\}_{n}=\sum_{k=1}^{n} d_{n}^{(k)}(\hat{r}, \hat{s}) y_{k}=(A y)_{n}$ for any

$$
n \in \mathbf{N} \text {. }
$$

From the relation (4.3), we observe that $f x=\left(f_{n} x_{n}\right) \in \ell_{1}$ whenever $x=\left(x_{k}\right) \in c_{0}(\hat{D}, p)$ if and only if $A y \in \ell_{1}$ whenever $y=\left(y_{k}\right) \in c_{0}(p)$. Hence, $f=\left(f_{n}\right) \in\left[c_{0}(\hat{D}, p)\right]^{\alpha}$ if and only if $A \in\left(c_{0}(p): \ell_{1}\right)$. Then, in equation (5.14), by considering $q_{n}=1$ for any $n \in \mathbf{N}$ we can write $f \in\left[c_{0}(\hat{D}, p)\right]^{\alpha}$ if and only if $\sup _{K \in \wp} \sum_{n}\left|\sum_{k \in K} a_{n k}\right| S^{\frac{-1}{p_{k}}}<\infty$ and consequently, $\left[c_{0}(\hat{D}, p)\right]^{\alpha}=T_{1}(p)$.

Consider the equality

$$
\sum_{k=1}^{n} f_{k} x_{k}=\sum_{k=1}^{n}\left(\sum_{j=1}^{k} d_{k}^{(j)}(\hat{r}, \hat{s}) y_{j}\right) f_{k}=(M y)_{n},
$$

where $M=\left(m_{n k}\right)$ is defined by

$$
n k=\left\{\begin{array}{c}
{ }_{j=1}^{k} d_{k}^{(j)}(\hat{r}, \hat{s}) f_{j}, \quad 1 \leq k \leq n \\
0, \quad k>n
\end{array}\right.
$$

for any $n, k \in \mathbf{N}$.

Now, from the relation (4.4), we observe that, $f x=\left(f_{n} x_{n}\right) \in c s$ whenever $x=\left(x_{k}\right) \in c_{0}(\hat{D}, p)$ if and only if $M y \in c s$ whenever $y=\left(y_{k}\right) \in c_{0}(p)$. Hence, $f=\left(f_{n}\right) \in\left[c_{0}(\hat{D}, p)\right]^{\beta}$ if and only if $M \in\left(c_{0}(p): c\right)$.

Again, we derive from the equation (5.10) and (5.11), by taking $q_{n}=1$ for any $n \in \mathbf{N}$ and some $S \in N_{2}$ we have $\sum_{k=0}^{n}\left|m_{n k}\right| S^{\frac{-1}{p_{k}}}<\infty$ and there exists scalar $\beta_{k} \in \mathbf{C}$ for any $k \in \mathbf{N}$ such that $\sup _{n \in \mathbf{N}}\left|\sum_{k=1}^{n} m_{m k}-\beta_{k}\right| S^{\frac{-1}{p_{k}}}<$ $\infty$, respectively, which implies that $\left[c_{0}(\hat{D}, p)\right]^{\beta}=T_{2}(p) \cap T_{3}(p)$. Now, we 
deduce from equation (5.9) that $f x=\left(f_{n} x_{n}\right) \in b s$ whenever $x=\left(x_{k}\right) \in$ $c_{0}(\hat{D}, p)$ if and only if $M y \in \ell_{\infty}$ whenever $y=\left(y_{k}\right) \in c_{0}(p)$. Hence, $f=\left(f_{n}\right) \in\left[c_{0}(\hat{D}, p)\right]^{\gamma}$ if and only if $M \in\left(c_{0}(p): \ell_{\infty}\right)$. Therefore, by equation (5.9) with $q_{n}=1$ for any $n \in \mathbf{N}$, we attain $\left[c_{0}(\hat{D}, p)\right]^{\gamma}=T_{4}(p)$.

\section{Matrix Transformations}

In summability theory, different classes of matrices have been investigated. Characterization of matrix classes is found in Rath and Tripathy [20], Tripathy and Sen [23] and many others.

Let $\lambda$ denote any of the sequence spaces $c_{0}, c$ or $\ell_{\infty}$ and $\mu$ be any given sequence space. In this section, we characterize the classes $(\lambda(\hat{D}, p): \mu)$ and $(\mu: \lambda(\hat{D}, p))$ of infinite matrices. Throughout we consider, $\hat{b}_{n k}=$ $\sum_{i=k}^{\infty} \frac{(-1)^{i} s_{i} s_{i+3}--s_{n-3}}{r_{i} r_{i+3}--r_{n}} b_{n i}$, for any $k, n \in \mathbf{N}$.

Theorem 5.1: Suppose that the elements of the infinite matrices $U=\left(u_{n k}\right)$ and $V=\left(v_{n k}\right)$ be connected with the relation

$u_{n k}=r_{k} v_{n k}+s_{k+3} v_{n, k+3}$ orv $_{n k}=\sum_{i=k}^{\infty} \frac{(-1)^{i} s_{i} s_{i+3}---s_{n-3}}{r_{i} r_{i+3}--r_{n}} u_{n i}$ forany $k, n \in \mathbf{N}$

and $\lambda$ be any of the spaces $c_{0}, c$ or $\ell_{\infty}$ and $\mu$ be any given sequence space.

Then, $U \in(\lambda(\hat{D}, p): \mu)$ if and only if

$$
V \in(\lambda(p): \mu) \text { and }
$$

$\mathrm{V}^{n} \in(\lambda(p): c)(5.2)$

for any fixed $n \in \mathbf{N}$, where $V^{n}=\left(v_{m k}^{(n)}\right)$ with

$$
v_{m k}^{(n)}=\left\{\begin{array}{l}
\sum_{i=k}^{m} \frac{(-1)^{i} s_{i} s_{i+3}---s_{n-3}}{r_{i} r_{i+3}---r_{n}} u_{n i}, \quad 0 \leq k \leq m \\
0, \quad k>m
\end{array}\right.
$$

for any $m, k \in \mathbf{N}$.

Proof: Suppose that the infinite matrices $U=\left(u_{n k}\right)$ and $V=\left(v_{n k}\right)$ be connected with the relation (5.1) and let $\mu$ be any given sequence space. One can easily prove that the spaces $(\lambda, p)$ and $\lambda(p)$ are paranorm isomorphic. 
Let $U \in(\lambda(\hat{D}, p): \mu)$ and $y \in \lambda(p)$. Then, $V \hat{D}(\hat{r}, 0,0, \hat{s})$ clearly exists and $\left(u_{n k}\right)_{k \in \mathbf{N}} \in[\lambda(\hat{D}, p)]^{\beta}$, which implies that (5.2) is necessary and $\left(v_{n k}\right)_{k \in \mathbf{N}} \in[\lambda(p)]^{\beta}$ for each $n \in \mathbf{N}$. Thus, $V y$ exists for all $y \in \lambda(p)$ and hence by letting $m \rightarrow \infty$ in the equality

$$
\sum_{k=1}^{m} u_{n k} x_{k}=\sum_{k=1}^{m}\left[\sum_{i=k}^{m} \frac{(-1)^{i} s_{i} s_{i+3}---s_{n-3}}{r_{i} r_{i+3}--r_{n}} u_{n i}\right] y_{k}
$$

Now, using (5.1) we have $U x=V y$, which concludes that $V \in(\lambda(p)$ : $\mu)$.

Conversely, let $V \in(\lambda(p): \mu)$ and suppose it holds the relation (5.2). Also let $y=\left(y_{k}\right) \in \lambda(p)$. Then, we have $\left(v_{n k}\right)_{k \in \mathbf{N}} \in[\lambda(p)]^{\beta}$, which gives together with the relation (5.2) that $\left(u_{n k}\right)_{k \in \mathbf{N}} \in[\lambda(\hat{D}, p)]^{\beta}$ for each $n \in \mathbf{N}$.

Thus, $U x$ exists and consequently, from the relation (5.3) by letting $m \rightarrow \infty$ we have $V y=U x$ and hence $U \in(\lambda(\hat{D}, p): \mu)$.

Theorem 5.2 Suppose that the elements of the infinite matrices $E=\left(e_{n k}\right)$ and $F=\left(f_{n k}\right)$ are connected with relation $f_{n k}=r_{n} e_{n k}+s_{n-3} e_{n-3, k}$ for any $k, n \in \mathbf{N}, \lambda$ is any of the spaces $c_{0}, c$ or $\ell_{\infty}$ and $\mu$ be any given sequence space. Then, $E \in(\mu: \lambda(\hat{D}, p))$ if and only if $F \in(\mu: \lambda(p))$.

Proof: Let $z=\left(z_{k}\right) \in \mu$ and consider the equality $\sum_{k=1}^{m} f_{n k} z_{k}=\sum_{k=1}^{m}\left(r_{n} e_{n k}+\right.$ $\left.s_{n-3} e_{n-3, k}\right) z_{k}$ for any $m, n \in \mathbf{N}$. Then by letting $m \rightarrow \infty$, we have $(F z)_{n}=$ $\{\hat{D}(\hat{r}, 0,0, \hat{s}) E z\}_{n}$ for any $n \in \mathbf{N}$. Hence, we observe that $E z \in \lambda(\hat{D}, p)$ whenever $z \in \mu$ if and only if $F z \in \lambda(p)$ whenever $z \in \mu$. This completes the proof.

Let $\left(q_{n}\right)$ be a non-decreasing bounded sequence of positive real numbers. Also, let $S$ and $T$ denote the natural numbers. Finally, the sets $K_{1}$ and $K_{2}$ are defined by

$K_{1}=\left\{k \in \mathbf{N}: p_{k} \leq 1\right\}$ and $K_{2}=\left\{k \in \mathbf{N}: p_{k}>1\right\}$.

The following lemmas are consider from Grosse-Erdmann[9] which gives the characterization of matrix mappings between Maddox's sequence spaces $\ell(p), c_{0}(p), c(p)$ and $\ell_{\infty}(p)$.

Lemma 5.3 Let $B=\left(b_{n k}\right)$ be an infinite matrix. Then, the following statements hold: 
(i) $B \in\left(\ell_{\infty}(p): \ell_{\infty}(q)\right)$ if and only if

$$
\forall S>1 \ni \sup _{n \in \mathbf{N}}\left(\sum_{k \in \mathbf{N}}\left|b_{n k}\right| S^{\frac{1}{p_{k}}}\right)^{q_{n}}<\infty,
$$

(ii) $B \in\left(\ell_{\infty}(p): c(q)\right)$ if and only if

$$
\forall S>1 \ni \sup _{n \in \mathbf{N}} \sum_{k \in \mathbf{N}}\left|b_{n k}\right| S^{\frac{1}{p_{k}}}<\infty,
$$

$\exists\left(\beta_{k}\right) \in w$ and

$$
\forall S>1 \ni \lim _{n \rightarrow \infty}\left(\sum_{k}\left|b_{n k}-\beta_{k}\right| S^{\frac{1}{p_{k}}}\right)^{q_{n}}=0,
$$

(iii) $B \in\left(\ell_{\infty}(p): c_{0}(q)\right)$ if and only if

$$
\forall S>1 \ni \lim _{n \rightarrow \infty}\left(\sum_{k}\left|b_{n k}\right| S^{\frac{1}{p_{k}}}\right)^{q_{n}}=0,
$$

(iv) $B \in\left(\ell_{\infty}(p): \ell(q)\right)$ if and only if $\sup _{K \in \wp} \sum_{n}\left|\sum_{k \in K} b_{n k} S^{\frac{1}{p_{k}}}\right|^{q_{n}}<\infty$

$$
\text { forany }
$$

$\mathrm{q}_{n} \geq 1$ and for any $S>1$. (5.8)

Lemma 5.4 Let $B=\left(b_{n k}\right)$ be an infinite matrix. Then, the following statements hold:

(i) $B \in\left(c_{0}(p): \ell_{\infty}(q)\right)$ if and only if

$$
\forall S>1 \ni \sup _{n \in \mathbf{N}}\left(\sum_{k \in \mathbf{N}}\left|b_{n k}\right| S^{\frac{-1}{p_{k}}}\right)^{q_{n}}<\infty,
$$

(ii) $B \in\left(c_{0}(p): c(q)\right)$ if and only if

$$
\forall S>1 \ni \sup _{n \in \mathbf{N}} \sum_{k \in \mathbf{N}}\left|b_{n k}\right| S^{\frac{-1}{p_{k}}}<\infty,
$$


$\forall T, \exists S>1 \ni \sup _{n \in \mathbf{N}} \sum_{k \in K_{2}}\left|b_{n k}-\beta_{k}\right| T^{\frac{1}{q_{n}}} S^{\frac{-1}{p_{k}}}<\infty,(5.11)$

$$
\exists\left(\beta_{k}\right) \in w \ni \lim _{n \rightarrow \infty}\left|b_{n k}-\beta_{k}\right|^{q_{n}}=0,
$$

(iii) $B \in\left(c_{0}(p): c_{0}(q)\right)$ if and only if (5.11)holds with

$$
\beta_{k}=0
$$

for any $k \in \mathbf{N}$ and $\lim _{n \rightarrow \infty}\left|b_{n k}\right|^{q_{n}}=0$ for each fixed $k \in \mathbf{N},(5.13)$

$($ iv $) B \in\left(c_{0}(p): \ell(q)\right)$ if and if

$$
\exists S>1 \ni \sup _{K \in \wp} \sum_{n}\left|\sum_{k \in K} b_{n k} S^{\frac{-1}{p_{k}}}\right|^{q_{n}}<\infty
$$

for any $q_{n} \geq 1$. (5.14)

Lemma 5.5 Let $B=\left(b_{n k}\right)$ be an infinite matrix. Then, the following statements hold: $(i) B \in(c(p): \ell(q))$ if and only if (5.9) holds and

$$
\sup _{n \in \mathbf{N}}\left|\sum_{k} b_{n k}\right|^{q_{n}}<\infty,
$$

(ii) $B \in(c(p): c(q))$ if and only if (5.10), (5.11) and (5.12) hold and

$$
\exists \beta \in \mathbf{C} \ni \lim _{n \rightarrow \infty}\left|\sum_{k} b_{n k}-\beta\right|^{q_{n}}=0,
$$

(iii) $B \in\left(c(p): c_{0}(q)\right)$ if and only if (5.13), holds and

$$
\begin{gathered}
\forall T, \exists S>1 \ni \sup _{n \in \mathbf{N}} \sum_{k \in K_{2}}\left|b_{n k}\right| T^{\frac{1}{q_{n}}} S^{\frac{-1}{p_{k}}}<\infty, \\
\lim _{n \rightarrow \infty}\left|\sum_{k} b_{n k}\right|^{q_{n}}=0,
\end{gathered}
$$

(iv) $B \in(c(p): \ell(q))$ if and only if (5.14) holds and

$$
\sum_{n}\left|\sum_{k} b_{n k}\right|^{q_{n}}<\infty
$$

for any $q_{n} \geq 1(5.19)$ 
Lemma 5.6 Let $B=\left(b_{n k}\right)$ be an infinite matrix. Then, the following statements hold:

(i) $B \in(\ell(p): c(q))$ if and only if (5.12) holds and

$$
\begin{gathered}
\sup _{n \in \mathbf{N}} \sup _{k \in K_{1}}\left|b_{n k}\right|^{p_{k}}<\infty, \\
\exists S>1 \ni \sup _{n \in \mathbf{N}} \sum_{k}\left|b_{n k} S^{-1}\right|^{p_{k}}<\infty,
\end{gathered}
$$

$\exists\left(\beta_{k}\right) \in w$ and $\forall T>1 \ni \sup _{n \in \mathbf{N}} \sup _{k \in K_{1}}\left(\left|b_{n k}-\beta_{k}\right| T^{\frac{1}{q_{n}}}\right)^{p_{k}}<\infty,(5.22)$

$\exists\left(\beta_{k}\right) \in w$ and $\forall T, \exists S>1 \ni \sup _{n \in \mathbf{N}} \sup _{k \in K_{2}}\left(\left|b_{n k}-\beta_{k}\right| T^{\frac{1}{q_{n}}} S^{-1}\right)^{p_{k}}<\infty,(5.23)$

(ii) $B \in\left(\ell(p): c_{0}(q)\right)$ if and only if (5.13) holds and

$\sup _{n \in \mathbf{N}} \sup _{k \in K_{1}}\left|a_{n k} T^{\frac{1}{q_{n}}}\right|^{p_{k}}<\infty, \forall T>1,(5.24)$

$\exists S>1 \ni \sup _{n \in \mathbf{N}} \sum\left|a_{n k} T^{\frac{1}{q_{n}}} S^{-1}\right|^{p_{k}}<\infty$ for any $T>1,(5.25)$

(iii) $B \in(\ell(p): \ell \infty(q))$ if and only if

$\exists T>1 \ni \sup _{n \in \mathbf{N}} \sup _{k \in K_{2}}\left|b_{n k} T^{\frac{-1}{q_{n}}}\right|^{p_{k}}<\infty$,

$\exists T>1 \ni \sup _{n \in \mathbf{N}} \sup _{k \in K_{2}}\left|a_{n k} T^{\frac{-1}{q_{n}}}\right|^{p_{k}}<\infty .(5.26)$

Now, the following we may quote our theorems without proof on the characterization of some matrix classes concerning with the sequence spaces $c_{0}(\hat{D}, p), c(\hat{D}, p)$ and $\ell_{\infty}(\hat{D}, p)$.

Theorem 5.7 Let $B=\left(b_{n k}\right)$ be an infinite matrix. Then, the following statements hold: 
(i) $B \in\left(c_{0}(\hat{D}, p): \ell(q)\right)$ if and only if (5.14) also holds with $\hat{b}_{n k}$ instead of $b_{n k}$ and (5.2) also holds with $\lambda=c_{0}$.

(ii) $B \in\left(c_{0}(\hat{D}, p): c(q)\right)$ if and only if (5.10), (5.11) and (5.12) hold with $\hat{b}_{n k}$ instead of $b_{n k}$ and (5.2) also holds with $\lambda=c_{0}$.

(iii) $B \in\left(c_{0}(\hat{D}, p): \ell_{\infty}(q)\right)$ if and only if (5.9) also holds with $\hat{b}_{n k}$ instead of $b_{n k}$ and (5.2) also holds with $\lambda=c_{0}$.

Theorem 5.8 Let $B=\left(b_{n k}\right)$ be an infinite matrix. Then, the following statements hold:

(i) $B \in(c(\hat{D}, p): \ell(q))$ if and only if (5.14) and (5.19) hold with $\hat{b}_{n k}$ instead of $b_{n k}$ and (5.2) also holds with $\lambda=c$.

(ii) $B \in(c(\hat{D}, p): c(q))$ if and only if (5.10), (5.11), (5.12) and (5.16) hold with $\hat{b}_{n k}$ instead of $b_{n k}$ and (5.2) also holds with $\lambda=c$.

(iii) $B \in\left(c(\hat{D}, p): \ell_{\infty}(q)\right)$ if and only if (5.9) and (5.15) also hold with $\hat{b}_{n k}$ instead of $b_{n k}$ and (5.2) also holds with $\lambda=c$.

Theorem 5.9 Let $B=\left(b_{n k}\right)$ be an infinite matrix. Then, the following statements hold:

(i) $B \in\left(\ell_{\infty}(\hat{D}, p): \ell_{\infty}(q)\right)$ if and only if (5.8) also holds with $b_{n k}$ replaced by $\hat{b}_{n k}$ and $(5.2)$ also holds with $\lambda=\ell_{\infty}$.

(ii) $B \in\left(\ell_{\infty}(\hat{D}, p): c_{0}(q)\right)$ if and only if (5.7) also holds with $b_{n k}$ replaced by $\hat{b}_{n k}$ and (5.2) also holds with $\lambda=\ell_{\infty}$.

(iii) $B \in\left(\ell_{\infty}(\hat{D}, p): c(q)\right)$ if and only if (5.5) and (5.6) hold with $b_{n k}$ replaced by $\hat{b}_{n k}$ and (5.2) also holds with $\lambda=\ell_{\infty}$.

(iv) $B \in\left(\ell_{\infty}(\hat{D}, p): \ell_{\infty}(q)\right)$ if and only if (5.4) also holds with $b_{n k}$ replaced by $\hat{b}_{n k}$ and (5.2) also holds with $\lambda=\ell_{\infty}$.

Theorem 5.10 Let $B=\left(b_{n k}\right)$ be an infinite matrix. Then, the following statements hold:

(i) $B \in\left(c_{0}(p): c(\hat{D}, q)\right)$ if and only if (5.10), (5.11) and (5.12) holds with $b_{n k}$ replaced by $v_{n k}$.

(ii) $B \in\left(c_{0}(p): \ell_{\infty}(\hat{D}, q)\right)$ if and only if (5.9) also holds with $b_{n k}$ replaced by $v_{n k}$. 
Theorem 5.11 Let $B=\left(b_{n k}\right)$ be an infinite matrix. Then, the following statements hold:

(i) $B \in(c(p): c(\hat{D}, q))$ if and only if (5.10), (5.11), (5.12) and (5.16) holds with $b_{n k}$ replaced by $v_{n k}$.

(ii) $B \in\left(c(p): \ell_{\infty}(\hat{D}, q)\right)$ if and only if (5.9) and (5.15) holds with $b_{n k}$ replaced by $v_{n k}$.

Theorem 5.12 Let $B=\left(b_{n k}\right)$ be an infinite matrix. Then, the following statements hold:

(i) $B \in\left(\ell_{\infty}(p): c_{0}(\hat{D}, q)\right)$ if and only if (5.7) also holds with $b_{n k}$ replaced by $v_{n k}$.

(ii) $) B \in\left(\ell_{\infty}(p): c(\hat{D}, q)\right)$ if and only if (5.5) and (5.6) holds with $b_{n k}$ replaced by $v_{n k}$.

(iii) $B \in\left(\ell_{\infty}(p): \ell_{\infty}(\hat{D}, q)\right)$ if and only if (5.4) also holds with $b_{n k}$ replaced by $v_{n k}$.

Theorem 5.13 Let $B=\left(b_{n k}\right)$ be an infinite matrix. Then, the following statements hold:

(i) $B \in\left(\ell(p): c_{0}(\hat{D}, q)\right)$ if and only if (5.13) and (5.24)-(5.25) holds with $b_{n k}$ replaced by $v_{n k}$

(ii) $B \in(\ell(p): c(\hat{D}, q))$ if and only if (5.12) and (5.20)-(5.23) holds with $b_{n k}$ replaced by $v_{n k}$.

(iii) $B \in\left(\ell(p): \ell_{\infty}(\hat{D}, q)\right)$ if and only if (5.26) and (5.27) holds with $b_{n k}$ replaced by $v_{n k}$.

\section{Conclusion:}

The spectrum of the matrix class $D(r, 0,0, s)$ has been investigated by Tripthy and Paul [22] is a special case of $\hat{D}(\hat{r}, 0,0, \hat{s})$ if we consider $\hat{r}=r e$ and $\hat{s}=s e$. The results investigated in this paper are more general. 


\section{References}

[1] B. Altay and F. Başar, "On the paranormed Riesz sequence spaces of non-absolute type", Southeast Asian Bulletin of Mathematics, vol. 26, no. 5, pp. 701-715, 2002. [On line]. Available: https:/ / bit.ly/ 3v8HRmQ

[2] B. Altay and F. Başar, "Some paranormed sequence spaces of nonabsolute type derived by weighted mean", Journal of mathematical analysis and applications, vol. 319, no. 2, pp. 494-508, 2006, doi: 10.1016/j.jmaa.2005.06.055

[3] B. Altay and F. Başar, "Certain topological properties and duals of the matrix domain of a triangle matrix in a sequence space", Journal of mathematical analysis and applications, vol. 336, no. 1, pp. 632-645, 2007, doi: 10.1016/j.jmaa.2007.03.007

[4] B. Altay and F. Başar, "The matrix domain and the fine spectrum of the difference operator $\Delta$ on the sequence space $\ell_{p},(0<p<1)$ ", Communications in mathematical analysis, vol. 2, no. 2, pp. 1-11, 2007.

[5] C. Aydin and B. Altay B, "Domain of generalized difference matrix B(r, s) on some Maddox's spaces", Thai journal of mathematics, vol. 11, no. 1, pp. 87-102, 2013. [On line]. Available: https:// bit.ly/2S08Wu7

[6] C. Aydin and F. Başar, "Some new paranormed sequence spaces", Information sciences, vol. 160, no. 1-4, pp. 27-40, 2004, doi: 10.1016/j.ins.2003.07.009

[7] F. Başar, B. Altay and M. Mursaleen, "Some generalizations of the space $b v_{p}$ of $p$-bounded variation sequences", Nonlinear analysis: theory, methods \& applications, vol. 68, no. 2, pp. 273-287, 2008, doi:10.1016/j.na.2006.10.047

[8] P. Chandra and B. C. Tripathy, "On generalized Kothe-Toeplitz duals of some sequence spaces", Indian journal of pure and applied mathematics, vol. 33, no. 8, pp. 1301-1306, 2002. [On line]. Available: https:// bit.ly/ 3yhBEqQ

[9] K-G. Grosseerdmann, "Matrix transformations between the sequence spaces of Maddox", Journal of mathematical analysis and applications, vol. 180, no. 1, pp. 223-238, 1993, doi: 10.1006/jmaa.1993.1398 
[10] A. M. Jarrah and E. Malkowsky, "BK spaces, bases and linear operators", Rendiconti del Circolo Matematico di Palermo Serie 2. Supplemento. vol. 52, pp. 177-191, 1998. [On line]. Available: https:/ / bit.ly/ 3tZTtr4

[11] M. Kiriş̧̧i and F. Başar, "Some new sequence spaces derived by the domain of generalized difference matrix", Computers \& mathematics with applications, vol. 60, no. 5, pp. 1299-1309, 2010, doi:10.1016/j.camwa.2010.06.010

[12] G. Köthe and O. Toeplitz, "Lineare Räume mit unendlich vielen Koordinaten und Ringe unendlicher Matrizen", Journal für die reine und angewandte Mathematik, vol. 171, pp. 193-226, 1934. [On line]. Available: https:/ / bit.ly/ 3huHLlP

[13] I. J. Maddox, "Paranormed sequence spaces generated by infinite matrices", Mathematical Proceedings of the Cambridge Philosophical Society, vol. 64, no. 2, pp. 335-340, 1968. https:// doi.org/ 10.1017/ S0305004100042894

[14] I. J. Maddox, "Some properties of paranormed sequence spaces", Journal of the London Mathematical Society, vol. 2-1, no. 1, pp. 316-322, 1969, doi: 10.1112/jlms/ s2-1.1.316

[15] I. J. Maddox, Infinite matrices of operators. Berlin: Springer, 1980, doi: 10.1007/ BFb0088196

[16] E. Malkowsky, "Recent results in the theory of matrix transformations in sequence spaces", Matematički vesnik, vol. 49, no. 209, pp. 187-196, 1997. [On line]. Available: https:/ / bit.ly/ 3u4E8pr

[17] E. Malkowsky and E.Savas, "Matrix transformations between sequence spaces of generalized weighted means", Applied mathematics and computation, vol. 147, no. 2, pp. 333-345, 2004, doi: 10.1016/ S0096-3003(02)00670-7

[18] P. N. Ng and P. Y. Lee, "Cesàro sequence spaces of non-absolute type”, Annales Societatis Mathematicae Polonae. Series 1: Commentationes mathematicae, vol. 20, no. 2, pp. 429-433, 1978. [On line]. Available: https:/ / bit.ly/ 33Yn6ON

[19] F. Özger and F. Başar, "Domain of the double sequential band matrix B $(\tilde{r}, \tilde{s})$ on some Maddox's spaces", Acta mathematica scientia, vol. 34, no.2, pp. 394-408, 2014, doi: 10.1016/ S0252-9602(14)60014-X

[20] D. Rath and B. C. Tripathy, "Matrix maps on sequence spaces associated with sets of integers", Indian journal of pure and applied mathematics, vol. 27, no. 2, pp. 197-206, 1996. [On line]. Available: https:/ / bit.ly/ 3w7EFrQ

[21] A. Sönmez, "Some new sequence spaces derived by the domain of the triple band matrix", Computers \& mathematics with applications, vol. 62, no. 2, pp. 641-650, 2011, doi: 10.1016/j.camwa.2011.05.045 
[22] B. C. Tripathy and A. Paul, "The spectrum of the operator D(r, 0, 0, s) over the sequence spaces c0 and c", Kyungpook mathematical journal, vol. 53, no. 2, pp. 247-256, 2013, doi: 10.5666/ KMJ.2013.53.2.247

[23] B. C. Tripathy and M. Sen, "Characterization of some matrix classes involving paranormed sequence spaces", Tamkang journal of mathematics, vol. 37, no.2, pp. 155-162, 2006, doi: 10.5556/ j.tkjm.37.2006.160 